\title{
Wall stress and deformation analysis in a numerical model of pulse wave propagation
}

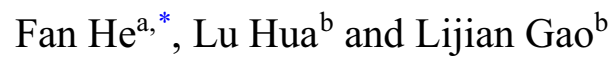 \\ ${ }^{a}$ Department of Mechanics, School of Science, Beijing University of Civil Engineering and \\ Architecture, Beijing, 100044, China \\ ${ }^{b}$ Key Laboratory of Clinical Trial Research in Cardiovascular Drugs, Ministry of Health, Fuwai \\ Hospital, National Center for Cardiovascular Diseases, Chinese Academy of Medical Sciences and \\ Peking Union Medical College, Beijing, 100037, China
}

\begin{abstract}
To simulate pulse wave propagation, we set up a wave propagation model using blood-wall interaction in previous work. In this paper, our purpose is to investigate wall stress and deformation of the wave propagation model. The finite element method is employed for solving the governing equations of blood and wall. Our results suggest that there are two peaks in the circumferential stress and strain distributions of the normal model. The stress and strain values change with the varieties of different factors, such as wall thickness and vessel diameter. The results indicate that different parameters of fluid and tube wall have remarked impact on wall stress and deformation.
\end{abstract}

Keywords: Wave propagation, finite element method, stress, deformation

\section{Introduction}

It is a common phenomenon that wave propagates in a tube. The wave propagation in arteries has a lot of specializations and is different from that in other tubes. Pulse waves have been also investigated widely as well as hemodynamics [1-5]. Pulse wave propagation in arteries is an important issue. It is a fact that non-destructive evaluation can be performed using pulse wave. Though pulse wave propagation has been investigated experimentally [6], it seems that it is little considered in numerical simulation.

The compliance of blood vessel wall makes blood flow and vessel wall interact each other. Blood flow dynamics induces the deformation of vessel wall, whereas the wall deformation affects blood flow. The advances of computer performance and numerical technologies make numerical simulation of fluid-structure interaction possible. The application background of fluid-structure interaction in arteries is to forecast and evaluate aneurysm rupture thoroughly from the viewpoint of both fluid and solid mechanics. The role of compliant arterial wall has been quite concerned. Thus, a simple method of finding the role is to compare with the condition of rigid wall. According to the method, the same

\footnotetext{
* Address for correspondence: Fan He, Department of Mechanics, School of Science, Beijing University of Civil Engineering and Architecture, Beijing, 100044, China. Tel.: +861061209221; Fax: +861061209221; E-mail: hefan@bucea.edu.cn.
} 
conclusion has been drawn by many researchers, that is, wall shear stress is decreased by the compliance of arterial wall through fluid-structure interaction [7-9].

Mechanical properties of arterial wall and hemodynamics have been investigated numerically through fluid-wall interaction [10-14]. The work of the blood-wall interaction mainly focuses on blood flow distributions and wall deformation. Li [15] investigated the flow field and stress field for different degrees of stenoses through fluid-structure interaction. Borghi [16] performed a fluid-solid interaction simulation in thoracoabdominal aneurysms to investigate the role of flow patterns and internal wall stress on the pathology. Khanafer [17] analyzed pulsatile turbulent flow characteristics in an axisymmetric aortic aneurysm model using fluid-structure interaction. The similar study could be found in the work of Tang [18], Wolters [19], Li [20] and Scotti [21].

The analysis of wall stress and deformation in pulse wave propagation is still lacking. The purpose of this paper is to investigate wall stress and deformation based on the constructed wave propagation model. The topic of pulse wave propagation and flow distributions has been included in our previous papers $[22,23]$.

\section{The mathematical model}

\subsection{Blood and wall models}

For the laminar, incompressible and Newtonian fluid assumption, the density and the dynamic viscosity of blood are constant. The typical values of $\rho=1050 \mathrm{~kg} / \mathrm{m}^{3}$ and $\mu=0.0035 \mathrm{~Pa} \cdot \mathrm{s}$ are used. The Young's modulus and the Poisson's ratio of elastic wall are set to be $5 \mathrm{MPa}$ and 0.499 . A rigidelastic-rigid model is set up, as shown in Figure 1. The elastic wall has a thickness of $2 \mathrm{~mm}$ and the blood domain has a diameter of $20 \mathrm{~mm}$. The each length of three segments is set to be $300 \mathrm{~mm}$.

\subsection{Boundary conditions}

We specify a time-dependent velocity profile at the inlet (Figure 2), a zero normal traction at the outlet and no-slip boundary conditions at the wall. According to the proposition of Weydahl and

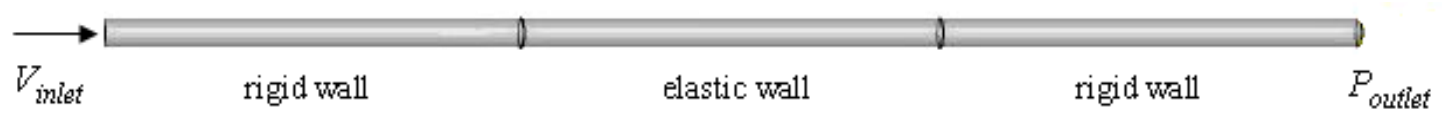

Fig. 1. The geometry of the model.

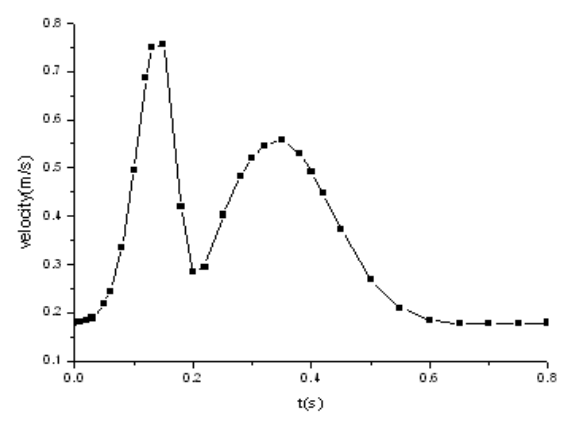

Fig. 2. The inlet velocity. 
Moore $\mathrm{Jr}$ [24] and $\mathrm{Lu}$, et al. [25], the initial conditions are set to be zero. One end of the blood vessel is entirely fixed and the other end is free. The velocities, displacements and normal stresses of fluid and wall at the fluid-wall interface should be guaranteed to be equal.

\subsection{Numerical implementation}

The blood-wall interaction algorithm based on a finite element method is constructed [22]. The whole domain of blood is discretized by 59370 four-node cells, and the wall is discretized by 9000 eight-node cells respectively. Numerical experiments are performed using more cells to evaluate grid independence. It is found that more cells do not induce obvious differences in results. We calculate three cycles with time interval of $0.01 \mathrm{~s}$ to achieve convergent and steady solutions. The details can be referred to our previous papers $[22,23]$.

\section{Results}

The wall stress and strain in two locations $(\mathrm{L}=360 \mathrm{~mm}$ and $540 \mathrm{~mm})$ are presented. $\mathrm{L}$ is the exact location away from the inlet.

\subsection{The normal model}

The model in Figure 1 is called as the normal model. The circumferential stress distributions in this model are shown in Figure 3(a). It can be seen that the stress distributions of different locations are almost identical. The stress values decrease gradually along the tube length. The wall stresses are caused by the fluid pressure. It is well known that circumferential stress and strain are directly related to the remodeling of arterial wall and affect the structure and physiological function of arterial wall. Therefore in the study the circumferential stresses and strains of the inner wall are concerned. The relationship between the stress and strain is linear-elastic. Thus the strain distributions are similar to the stress distributions (Figure 3(b)).

\subsection{The thickened wall model}

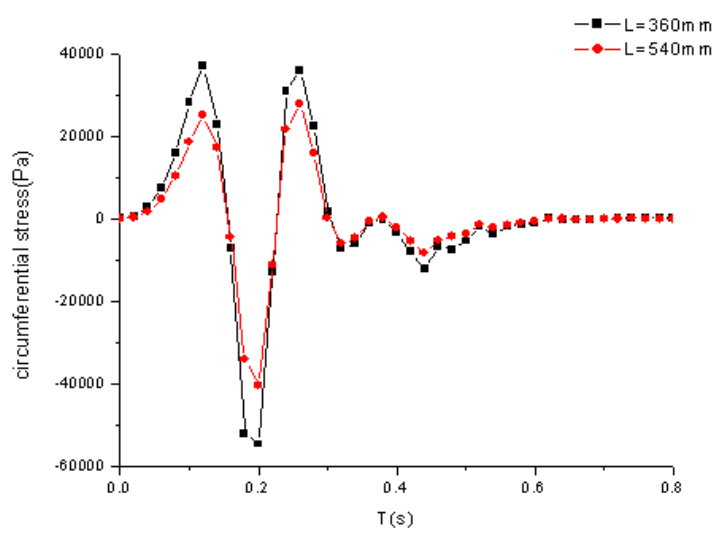

(a)

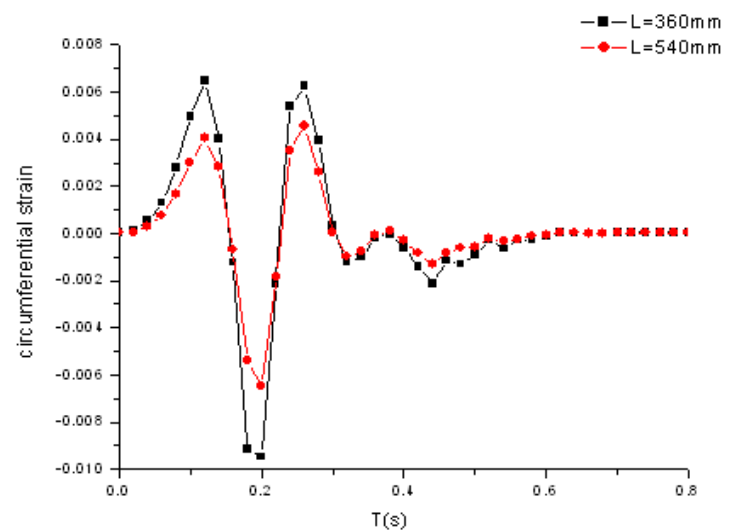

(b)

Fig. 3. The circumferential (a) stress and (b) strain distributions in the normal model. 
Based on the normal model, we have an increase of $2 \mathrm{~mm}$ in wall thickness in order to study the effects of elastic wall on stress and deformation. Thus, the new wall thickness is $4 \mathrm{~mm}$. It is seen that the circumferential stress distributions do not change in contrast to the normal model (Figure 4(a)). But the stress peak values are lower while the stress bottom values are higher. Thus the stress amplitude is narrower. Similarly, the strain amplitude is also narrower (Figure 4(b)).

\subsection{The thinned vessel model}

The vessel diameter is reduced from $20 \mathrm{~mm}$ to $16 \mathrm{~mm}$ but the wall thickness keeps constant at the meantime. Thus, the thinned vessel model is obtained. Compared with the normal model, the decrease of the vessel diameter does not also change the circumferential stress distributions (Figure 5(a)). However the stress peak values are lower while the stress bottom values are higher. As a result, the stress amplitude is narrower. The strain amplitude is likewise narrower (Figure 5(b)).

\section{Discussion}

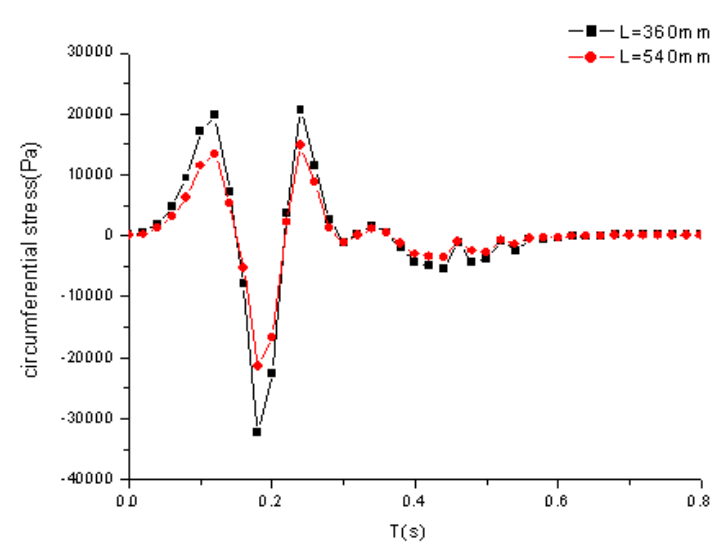

(a)

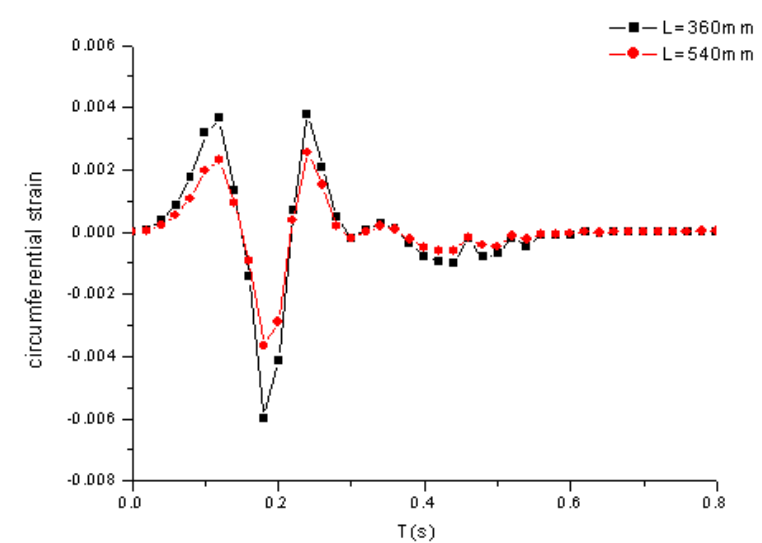

(b)

Fig. 4. The circumferential (a) stress and (b) strain distributions in the thickened wall model.

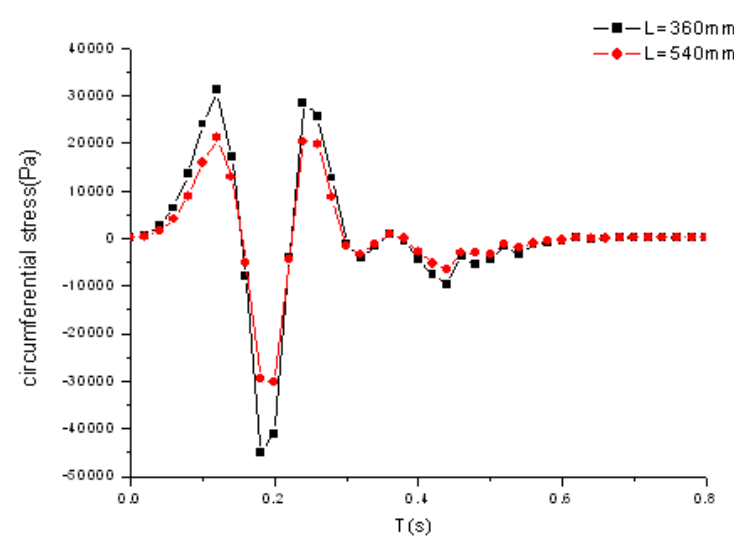

(a)

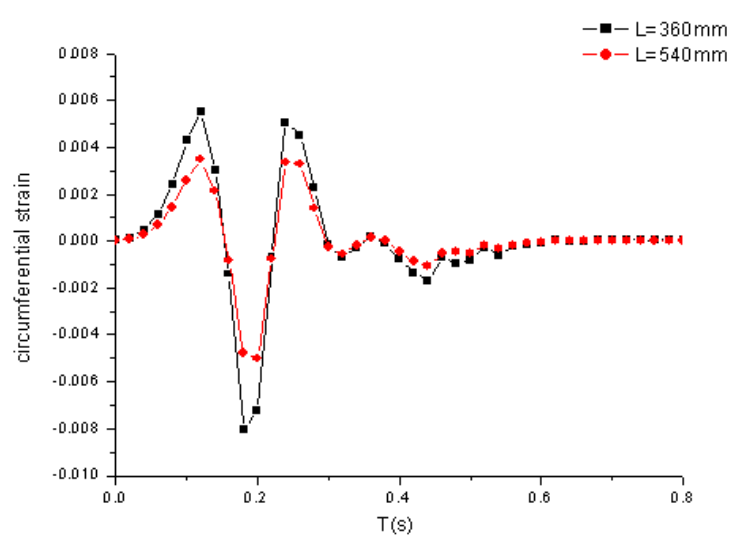

(b)

Fig. 5. The circumferential (a) stress and (b) strain distributions in the thinned vessel model. 
Under the normal condition, it is found that there are two peaks in the circumferential stress distributions. Despite of the conditions, the stress values decrease gradually along the tube length. The stress and strain distributions are not changed by the varieties of wall thickness and vessel diameter. However the stress values are significantly changed by different influence factors. The strain distributions are the same as the stress distributions. Therefore the strain values also change when the influence factors alter. It is found that the characteristics of wall stress and deformation change with variation of properties of arterial wall and blood. These results are consistent with experimental observation [26]. Hence, the changes of wall stress and deformation may cause abnormal arterial tissue and structure. The changes of these factors such as wall thickness and vessel diameter are linked to the relevant pathologies. Therefore they have actual significations. In this model, rigid parts are used at the two ends in order to simulate the process of wave propagation. Also, since the blood flow imposes large pulse pressures on the wall, large deformation by hyperelasticity is better to be used to model the change. The vessel wall is also found to be anisotropic with two families of fibers inside. These factors will be considered in our future work.

\section{Conclusions}

Wall stress and deformation in the wave propagation model have been performed numerically. Effects of parameters of tube wall and fluid on wall stress and deformation have been investigated and discussed in detail. The stress and strain values change with the varieties of different factors, such as wall thickness and vessel diameter. The results suggest that different parameters of fluid and tube wall have remarked impact on wall stress and deformation.

\section{Acknowledgments}

We thank National Natural Science Foundation of China (81401492), the Science and Technology Project of Beijing Municipal Commission of Education (KM201510016012) for financially supporting this research. The work is also supported by the Foundation of Research and Innovation Team (PXM 2013_014210_000173) and the Academic Innovation Team of Beijing University of Civil Engineering and Architecture (21221214111).

\section{References}

[1] D. Ku, D. Giddens, C. Zarins and S. Glagov, Pulsatile flow and atherosclerosis in the human carotid bifurcation: positive correlation between plaque and low and oscillating shear stress, Arteriosclerosis 5 (1985), 293-302.

[2] R.M. Nerem, Vascular fluid mechanics, the arterial wall and atherosclerosis, Journal of Biomechanical Engineering 114 (1992), 274-282.

[3] R. Ross, The pathogenesis of atherosclerosis: A perspective for the 1990s, Nature 362 (1993), 801-809.

[4] O. Araim, A.H. Chen and B. Sumpio, Hemodynamic forces: effects on atherosclerosis, New Surgery 1 (2001), 92-100.

[5] T.G. Papaioannou, E.N. Karatzis, M. Vavuranakis, J.P. Lekakis and C. Stefanadis, Assessment of vascular wall shear stress and implications for atherosclerotic disease, International Journal of Cardiology 113 (2006), 12-18.

[6] A.W. Khir and K.H. Parker, Measurements of wave speed and reflected waves in elastic tubes and bifurcations, Journal of Biomechanics 35 (2002), 775-783.

[7] Y. Liu, Y. Lai, A. Nagaraj, B. Kane, A. Hamilton, R. Greene, D.D. McPherson and K.B. Chandran, Pulsatile flow simulation in arterial vascular segments with intravascular ultrasound images, Medical Engineering \& Physics 23 (2001), 583-595. 
[8] M.S. Moayeri and G.R. Zendehbudi, Effects of elastic property of the wall on flow characteristics through arterial stenoses, Journal of Biomechanics 36 (2003), 525-535.

[9] N.M. Maurits, G.E. Loots and A.E.P. Veldman, The influence of vessel wall elasticity and peripheral resistance on the carotid artery flow wave form: A CFD model compared to in vivo ultrasound measurements, Journal of Biomechanics 40 (2007), 427-436.

[10] K. Perktold and G. Rappitsch, Computer simulation of local blood flow and vessel mechanics in a compliant carotid artery bifurcation model, Journal of Biomechanics 28 (1995), 845-856.

[11] D. Tang, C. Yang and D.N. Ku, A 3-D thin-wall model with fluid-structure interactions for blood flow in carotid arteries with symmetric and asymmetric stenoses, Computers and Structures 72 (1999), 357-377.

[12] E.S. Di Martino, G. Guadagni, A. Fumero, G. Ballerini, R. Spirito, P. Biglioli and A. Redaelli, Fluid-structure interaction within realistic three-dimensional models of the aneurysmatic aorta as a guidance to assess the risk of rupture of the aneurysm, Medical Engineering \& Physics 23 (2001), 647-655.

[13] A. Valencia and F. Solis, Blood flow dynamics and arterial wall interaction in a saccular aneurysm model of the basilar artery, Computers and Structures 84 (2006), 1326-1337.

[14] X.L. Yang, Y. Liu and J.M. Yang, Fluid-structure interaction in a pulmonary arterial bifurcation, Journal of Biomechanics 40 (2007), 2694-2699.

[15] M.X. Li, J.J. Beech-Brandt, L.R. John, P.R. Hoskins and W.J. Easson, Numerical analysis of pulsatile blood flow and vessel wall mechanics in different degrees of stenoses, Journal of Biomechanics 40 (2007), 3715-3724.

[16] A. Borghi, N.B. Wood, R.H. Mohiaddin and X.Y. Xu, Fluid-solid interaction simulation of flow and stress pattern in thoracoabdominal aneurysms: A patient-specific study, Journal of Fluids and Structures 24 (2008), 270-280.

[17] K.M. Khanafer, J. L. Bull and R. Berguer, Fluid-structure interaction of turbulent pulsatile flow within a flexible wall axisymmetric aortic aneurysm model, European Journal of Mechanics B/Fluids 28 (2009), 88-102.

[18] D. Tang, C. Yang, H. Walker, S. Kobayashi and D.N. Ku, Simulating cyclic artery compression using a 3D unsteady model with fluid-structure interactions, Computers and Structures 80 (2002), 1651-1665.

[19] B.J.B.M. Wolters, M.C.M. Rutten, G.W.H. Schurink, U. Kose, J. de Hart and F.N. van de Vosse, A patient-specific computational model of fluid-structure interaction in abdominal aortic aneurysms, Medical Engineering \& Physics 27 (2005), 871-883.

[20] Z. Li and C. Kleinstreuer, Fluid-structure interaction effects on sac-blood pressure and wall stress in a stented aneurysm, Journal of Biomechanical Engineering 127 (2005), 662-671.

[21] C.M. Scotti and E.A. Finol, Compliant biomechanics of abdominal aortic aneurysms: A fluid-structure interaction study, Computers and Structures 85 (2007), 1097-1113.

[22] F. He, Wave propagation in an elastic tube: A numerical study, International Journal of Computational Fluid Dynamics 27 (2013), 164-172.

[23] F. He, Wall shear stresses in a fluid-structure interaction model of pulse wave propagation, Journal of Mechanics in Medicine and Biology 14 (2014), 1450019.

[24] E.S. Weydahl and J.E. Moore Jr, Dynamic curvature strongly affects wall shear rates in a coronary artery bifurcation model, Journal of Biomechanics 34 (2001), 1189-1196.

[25] Y.L. Lu, X.Y. Lu, L.X. Zhuang and W. Wang, Breaking symmetry in non-planar bifurcation: Distribution of flow and wall shear stress, Biorheology 39 (2002), 431-436.

[26] T.J. Pedley, The Fluid Mechanics of Large Blood Vessels, Cambridge University Press, London, 1980. 